\title{
Infant Sleep Location: Bed Sharing, Room Sharing and Solitary Sleeping at 6 and 12 Weeks Postpartum
}

\author{
Robyn Stremler $^{1,2, *}$, Ellen Hodnett ${ }^{1}$, Laura Kenton ${ }^{1}$, Kathryn Lee ${ }^{3}$, James Macfarlane ${ }^{2}$, Shelly \\ Weiss $^{2}$, Julie Weston ${ }^{1}$ and Andrew Willan ${ }^{2}$ \\ ${ }^{1}$ Lawrence S. Bloomberg Faculty of Nursing, University of Toronto, Toronto, Canada \\ ${ }^{2}$ The Hospital for Sick Children, Toronto, Canada \\ ${ }^{3}$ School of Nursing, University of California, San Francisco, San Francisco, USA
}

\begin{abstract}
Objectives: Parents may have their infant sleep with them in the same bed (bed sharing [BS]), in the same room, but a separate bed (room sharing [RS]), or a separate room in a separate bed (solitary sleeping [SS]); prevalence estimates of these choices are limited. Little is known regarding the effects of infant sleep location (ISL) on infant or maternal sleep or other health outcomes.

Methods: Healthy first-time mothers $(\mathrm{n}=246)$ in an RCT of a sleep intervention provided information regarding planned and actual ISL at 6 and 12 weeks postpartum when maternal and infant sleep were measured using actigraphy. Other outcomes included subjective maternal sleep disturbance, breastfeeding exclusivity, fatigue, and depressive symptomatology.

Results: Most women (65\%) planned RS with their infant; the next most common plan was to use both BS and RS (22\%). The most common usual ISL was RS, with $46 \%$ of infants RS at 6 weeks and $39 \%$ at 12 weeks. Usual BS was common; at 6 weeks $17 \%$ of families were usually BS and $12 \%$ at 12 weeks. BS to any extent was quite common at $6(51 \%)$ and 12 weeks (41\%) postpartum. At 6 weeks, usually BS mothers had shorter stretches of sleep than those usually SS (130 mins vs 156 mins ; $\mathrm{p}=0.03$ ) and more awakenings than those usually RS and SS (11.2 vs 8.9 vs $8.3 ; \mathrm{p}=0.001)$. At 12 weeks, mothers who were usually RS had shorter stretches of sleep than those usually SS (164 mins vs 192 mins; $p=0.04)$. There were no significant differences between ISL groups on any other outcomes.

Conclusion: There is variation in ISL choice, and ISL changes across the first three months postpartum. Given our findings that ISL has effects on objective maternal sleep outcomes, further prospective, longitudinal research on the effects of ISL on a broad range of health outcomes for infant, mother and partner across the first postpartum year is needed.
\end{abstract}

Keywords: Sleep, parent, infant, bedsharing, cosleeping, actigraphy.

\section{INTRODUCTION}

Deciding where one's newborn will sleep is an issue faced by all families. New parents may choose to have their infant sleep with them in the same bed (bed sharing [BS]), in the same room, but in a separate bed (room sharing [RS]), or in a separate room in a separate bed (solitary sleeping [SS]). The American Academy of Pediatrics (AAP) [1] and the Canadian Pediatric Society (CPS) [2] recommend that parents practice RS for the first year and six months of an infant's life, respectively, and avoid BS and SS, as a means to decrease the risk of Sudden Infant Death Syndrome (SIDS) or other sleep-related deaths (e.g. due to suffocation, asphyxia, entrapment of the infant). These recommendations have generated debate regarding the relative risks and benefits of various infant sleep locations (ISL) and have

*Address correspondence to this author at the Lawrence S. Bloomberg Faculty of Nursing, University of Toronto, 155 College Street, Toronto, Ontario, M5T 1P8: Canada; Tel: 416-978-6925; Fax: 416-946-8681;

E-mail: robyn.stremler@utoronto.ca highlighted the wide variation in societal, cultural, and individual beliefs and practices around ISL [3, 4]. Although estimates of the prevalence of BS, RS or SS in the United States and Canada are limited, the AAP and CPS recommendations on ISL likely do not reflect the practices of all parents in North America.

Recent large scale $(n \geq 1800)$ survey data from the United States indicate that in the first three months of an infant's life BS occurred to some extent in $42-76 \%$ of families [5-7], with $14-44 \%$ always or almost always BS $[5,6,8]$. Rates of BS typically declined over time in those studies that surveyed women over the first year of the infant's life, with $27 \%$ practicing any BS at 12 months in one survey [5], and $13 \%$ BS most of the night at 6 months in another [8]. Perhaps because of the relatively recent introduction of recommendations around RS, few researchers have asked specifically about RS or SS rates, making it difficult to separate the two within those families who are not BS. In one study, rates of SS were seen to increase over the first year of the infant's life from $15 \%$ at 2 weeks to $71 \%$ at 12 
months [5]. A survey in urban US centers of 708 low income mothers of infants ( $<8$ months) indicated that $49 \%$ had practiced predominantly RS on the previous night [9].

One study provides Canadian estimates of ISL prevalence. Mothers of 3-4 month old infants in Manitoba were sent questionnaires; 293 surveys were completed $(26 \%$ return rate). Although $89 \%$ of women agreed that sleeping with one's baby had risks associated with the practice, $72 \%$ reported at least some BS with $43 \%$ reporting its use $>3$ times per week. One third of women who practiced BS did so for the entire night [10].

A number of maternal and infant characteristics have been associated with ISL, although evidence is conflicting and comparisons are limited to BS versus all other ISL. Breastfeeding is consistently associated with BS in Canada, US and England [5, 6, 8, 10, 11]. BS has been shown to be more likely among women of low socioeconomic status (SES) [5-8]. However, these studies did not determine the influence of factors such as lack of space in the home, or ability to purchase a crib. Single women [5-8], Hispanic [6, 9], Black [6, 7], and Asian/Pacific Islander [6, 7] families and those in crowded housing [8] have also been shown more likely to practice BS. However, in the UK, BS was not related to younger age of the mother, single motherhood or larger families; in fact those more socially advantaged were more likely to practice BS $[11,12]$. Similarly, a small sample of educated, moderate-high income families in an urban area in the US found no difference in BS rates across ethnic groups and high rates of BS in the first month of life [13].

\section{EFFECTS OF ISL ON SLEEP}

Caring for an infant, whose sleep is not yet consolidated at night, means that postpartum women and their partners experience both sleep deprivation and sleep fragmentation and report significant fatigue $[14,15]$. Chronic sleep deprivation and fragmentation significantly increase the risk of mood disorders, lapses in cognitive function and decreased well-being $[16,17]$. The effects of sleep deprivation and sleep fragmentation, so common in the postpartum, have important effects on multiple dimensions of health, yet we know little about how parents' sleep is affected by ISL.

Sleep in postpartum families has implications for infants as well; sleep problems in older children often persist from infancy [18]. While the effects of sleep loss are difficult to measure in infants due to their inability to respond to standardized testing approaches, furthering our understanding of the effects of ISL on infant sleep is essential to promotion of infant sleep and avoidance of persistent sleep problems. Of course, infant sleep and parent sleep in the postpartum are inextricably linked. Determining if ISL has effects on sleep for families could lead to interventions and health promotion strategies to improve families' sleep.

Most of what is known about the relationship of ISL to infant and maternal sleep is drawn from a study of 35 breastfeeding, Latino women, 20 of whom were routinely ( $\geq 4$ hours/night, $\geq 5$ days/week) BS, and 15 of whom were routinely SS ( $\leq 1$ night/week of BS) [19]. The women and their infants spent two nights in a sleep lab during which time their sleep was recorded using polysomnography. On one night the mother-infant pairs slept in their usual sleep conditions and on the other, they slept in the alternate condition; order of nights was randomly assigned. On the BS night, all mothers spent more time and a larger portion (4\% more) of the night in Stage 1-2 (lighter stages) sleep and less time and a smaller portion of the night (4\% less) in Stage 3-4 (deeper stages) sleep, regardless of usual ISL. There was no change in amount of REM sleep. Arousals from sleep to wake were more frequent during BS regardless of usual ISL (mean increase of 3.6 arousals per hour), however, total time awake at night did not increase, indicating that the arousals were brief. Sleep parameters were within the normal range, but given that these changes in sleep architecture occurred regardless of habitual ISL women may not "get accustomed" to BS. Women who practiced routine SS rated their sleep quality as lower on the BS night, so occasional BS may be more disruptive to subjective evaluations of sleep quality than routine BS. Synchronous arousals with the infant were also more frequent on the BS nights. An examination of the infant sleep data revealed that BS facilitated more arousals from Stage 3-4 sleep, a time when infant arousability is usually decreased [20]. On BS nights, infants had significantly greater duration and percentage of Stage 1-2 sleep and lower percentage of Stage 3-4 sleep than on the SS night with shorter episodes of Stage 3-4 sleep, regardless of usual ISL. Usually BS infants spent 21 minutes less time in Stage 3-4 on their BS night compared to the SS night. No differences in REM sleep were noted [20].

Two other studies have examined ISL as it relates to infant sleep. Video somnography of 9 usually BS and 9 usually SS infants in their usual sleep conditions was conducted at 6,9 and 12 months of age [21]. The routinely BS infants had significantly more awakenings at night; 5.37.5 per night versus 2.3-3.6 per night in SS infants. Although the BS infants woke more often, their awakenings were shorter in duration so the total amount of time awake at night did not differ between groups. DeLeon and Karraker found that among 41 9-month old infants, BS with a parent was associated with more time awake at night as reported by parents [22].

\section{EFFECTS OF ISL ON OTHER HEALTH OUTCOMES}

If ISL affects infant or parent sleep, it may also affect other important health outcomes. Since unrelieved postpartum fatigue and sleep disruption are factors in the development of postpartum depression [23-25], and sleep disturbance and insomnia are known risk factors for general depression in men and women [26, 27] the effects of ISL on fatigue and depression merit exploration. Routinely BS women are more likely to breastfeed, but this association does not demonstrate causation; certainly there are many successful breastfeeding dyads who are not routinely BS. No studies have examined the relationship between ISL and breastfeeding duration or exclusivity, perhaps the most important infant feeding outcomes.

Given the lack of estimates of ISL prevalence, especially since the 2004/2005 CPS/AAP recommendations promoting $\mathrm{RS}$, more data related to ISL practices are sorely needed. Our randomized, controlled trial (RCT) of a sleep intervention 
for women and their newborns provided us the opportunity to investigate ISL and its effects on maternal and infant sleep and other health outcomes in the early postpartum. The objectives of this secondary analysis were to examine: 1) plans for ISL in the immediate postpartum; 2) prevalence of BS, RS and SS at 6 and 12 weeks postpartum; and 2) effects of ISL on health outcomes including objective sleep (infant and maternal), subjective maternal sleep disturbance, breastfeeding exclusivity, maternal fatigue, and maternal depressive symptomatology at 6 and 12 weeks postpartum.

\section{MATERIALS AND METHODOLOGY}

\section{Sample}

Data from women $(n=246)$ enrolled in an RCT of a behavioral-educational sleep intervention [28] were used in this secondary analysis. The initial RCT was designed to determine the effect of a sleep intervention in the early postpartum on maternal sleep, infant sleep, depressive symptomatology, maternal fatigue, and exclusive breastfeeding rates at 6 and 12 weeks postpartum. The postpartum units of two university-affiliated hospitals (one Level III with 3600 births/year; one Level II with 2500 births/year) participated in the study.

Longitudinal mixed model analyses revealed no statistically significant group differences in any outcomes in the primary analysis, so data from the intervention and control group were aggregated for the secondary analysis related to ISL.

\section{Procedures for the TIPS Trial}

Research ethics board approval was obtained prior to beginning recruitment. Research assistants met with eligible women on the postpartum unit and obtained written informed consent from those willing to participate. Women (single or partnered) were included who: had given birth for the first time; had a singleton baby born at gestational age (GA) $\geq 37$ weeks; had a baby described as healthy in the newborn examination; lived in the greater Toronto area; and planned to provide fulltime infant care for at least the first 12 weeks after birth. Women with previous miscarriages at $<20$ weeks GA were eligible if the most recent pregnancy resulted in a first child. Women were excluded if they or their partners: had experienced stillbirth or perinatal death at $\geq 20$ weeks GA; had children from another relationship; used drugs or alcohol beyond social use; had a diagnosed sleep disorder; or were working night shifts. Women were also excluded if they: experienced postpartum complications or had an infant requiring prolonged hospital stay; had a poorly controlled chronic illness; used medications that affect sleep; were unable to read or understand English; or had no telephone in the home.

Consenting women had baseline data (e.g. birth weight, mode of delivery) collected from their medical charts, filled out a brief questionnaire (e.g. plans to bed-share with infant, depression score), and were randomized.

\section{Sleep Intervention Group}

As soon as possible after randomization, on the postpartum unit, the sleep intervention (SI) nurse delivered the in-hospital portion of the behavioral-educational intervention. The sleep intervention was a 45-minute, one-toone meeting where strategies to improve mother and baby sleep were discussed; no advice was given related to ISL. The mother also received a 20-page booklet elaborating on these tips. The advice given to the experimental group respected individual family choices related to ISL and infant feeding decisions. Mothers were contacted by the SI nurse 1, 2 , and 4 weeks after hospital discharge, content of the inhospital information session was reinforced, and any related questions were answered.

\section{Control group}

Immediately after randomization, the RA briefly met with the mother to inform her of her assignment to the control group. Women assigned to the control group received a phone call from the same RA at weeks 1,2 and 4; the RA inquired about the woman's and her baby's wellbeing, but did not offer any advice or support related to maternal or infant sleep. If women asked the RA for information related to sleep, the RA responded that $\mathrm{s} /$ he was not trained to provide such information.

\section{6 and 12 Week Follow-Up Home Visits}

At 6 and 12 weeks all mothers were visited in their homes by an RA and actigraphy was performed over 4 consecutive days and nights. The participant completed a sleep diary throughout the day and each morning and evening that she and her baby wore their actigraphs. The RA returned to the mother's home the day after the fourth night of actigraphy to collect the actigraphs and sleep diary data and to have the mother complete a short, self-administered questionnaire. Women were given a \$25 gift certificate at data collection completion at each of 6 and 12 weeks, in recognition of their time and commitment.

\section{Measures}

For the secondary analysis of ISL data, the following variables were examined.

\section{Planned ISL}

Data were gathered from the baseline questionnaire completed in the immediate postpartum, in-hospital. Women were asked to report if they planned to have their baby sleep in their bed at any time in the first 12 weeks postpartum, and if they planned to have their baby sleep in his/her own bed but in the mother's bedroom at any time in the first 12 weeks postpartum.

\section{Actual ISL}

Data were gathered from postpartum questionnaires at 6 and 12 weeks postpartum when women were asked "does your baby usually sleep with you in your bed?" and "does your baby usually sleep in your room but in his/her own bed?" Possible responses were "the whole night", "most of the night", "a small part of the night", and "never". Women were classified as Usually BS, Usually RS and Usually SS if they responded "the whole night" or "most of the night". Classifications were checked against women's response to "did your baby sleep in your bed with you last night?" on sleep diaries. Few classifications did not match diary responses (14 cases at 6 weeks; 10 cases at 12 weeks); given that the diary question did not specify the amount of time 
spent with the baby in bed classifications that did not match were not changed. The few cases classified as both Usually BS and Usually RS ( 7 cases at 6 weeks; 9 cases at 12 weeks) were set to missing.

Women were further classified as participating in any BS at 6 and 12 weeks postpartum. Any BS was defined as any response except "never" to "does your baby usually sleep with you in your bed?" on the questionnaire or stating that baby slept in bed with mother on at least one of the diary nights.

\section{Factors that Might Influence ISL}

On the 6 and 12 week postpartum questionnaires women were asked if they received any help looking after their baby at night that allowed them to stay in bed, and the number of other adults living in the home.

\section{Maternal and Infant Sleep Outcomes}

Data were gathered by actigraphy at 6 and 12 weeks postpartum and included maternal and infant: nocturnal (9pm-9am) sleep (minutes); longest nocturnal sleep period (minutes); daytime (9am-9pm) sleep (minutes); longest daytime sleep period (minutes); and number of nocturnal awakenings. Sleep data were averaged over four nights of data collection at each time point.

Actigraphy is a portable method for recording sleep data, conducted by use of an actigraph. The actigraph detects and records continuous motion data with a battery-operated wristwatch-size microprocessor that senses motion with a piezo-electric linear accelerometer. These detected movements are translated into digital counts across 1-minute intervals and stored in internal memory. Data from the actigraph are downloaded to a computer and interpreted using autoscoring programs. The Standards of Practice Committee of the American Academy of Sleep Medicine have recommended at least 4 consecutive 24-hour periods of actigraph recording time [29]. For adults, an actigraph is worn comfortably around the wrist (weight approximately $60 \mathrm{~g}$ ), similar to a wristwatch and is left in place for the measurement period to detect motion over that period. The actigraph is water-resistant, but cannot be worn while bathing or swimming. For infants, the actigraph is worn around the ankle (weight approximately $25 \mathrm{~g}$ ), over a sock. Women began wearing Basic Motionlogger ${ }^{\circledR}$ (Ambulatory Monitoring Inc.) and infants wore Micro MiniMotionlogger $^{\circledR}$ (Ambulatory Monitoring Inc.) actigraphs on the Monday closest to when the infant achieved 6 and 12 weeks of age and wore them continuously until they were picked up on Friday; this approach avoids data recording on weekends when young adults significantly alter their sleep times. Action4 Software (Ambulatory Monitoring Inc.) was used for actigraphic data analysis with infant (Sadeh) and adult (Cole-Kripke) algorithms.

In addition, the maternal-infant sleep diary was used to support the actigraphy data and to provide data related to bed and wake times, and the number of nocturnal maternal awakenings noted by the mothers. For example, if a mother forgot to press the event marker on her actigraph when she went to bed or got up, her sleep diary was used to verify the correct times. Diary data were also used to determine that periods of complete inactivity recorded by actigraphy corresponded to when the mother recorded that actigraphs were removed (e.g. for bathing). Artifacts in actigraphy recording may result due to motion of the individual while asleep, for example from a moving vehicle, baby swing or stroller. Women were asked to record when their infant was asleep but "in motion" so that those times could be matched with rhythmic motion on the actigraphy recording. When sleep diary and actigraphy data indicated that artifacts were present data were recoded appropriately using Action4 software.

\section{Fatigue}

Morning fatigue was collected on the sleep diaries at 6 and 12 weeks postpartum by the Fatigue Visual Analogue Scale (Fatigue-VAS) [30]. The 7-item Fatigue-VAS consists of $100 \mathrm{~mm}$ visual analog lines; the line anchors are adjectives that relate to level of perceived fatigue (e.g. not at all tired to extremely tired). This shorter version of the Fatigue-VAS has good internal consistency reliability $($ Cronbach alpha coefficient $=0.94)$ [31]. Women completed this measure upon awakening along with their sleep diary during actigraphy data collection.

\section{Sleep Disturbance}

Sleep disturbance was measured using the General Sleep Disturbance Scale (GSDS) at baseline and 6 and 12 weeks; the GSDS is a 21-item scale that assesses components of subjective sleep disturbance including sleep initiation, sleep maintenance, perceived quality of sleep and maintenance of wakefulness [32]. Frequency of difficulties in the past week is rated along each dimension using an 8-point Likert scale (total score range 0-147) from "not at all" to "everyday". Internal consistency for the scale is good (Cronbach alpha reliability coefficient $=0.88$ ). A score above 42 for the total scale indicates a "poor sleeper". At 6 and 12 weeks postpartum women were also asked if they considered either their own or their baby's sleep to be a "problem".

\section{Depressive Symptoms}

To examine depressive symptomatology, the Edinburgh Postnatal Depression Scale (EPDS) [33] was administered at baseline, 6 and 12 weeks. Ten items inquire about maternal mood in the past 7 days and are rated on a 4-point scale (total score range 0-30); higher scores indicate lower maternal mood. The EPDS is the most frequently used instrument to assess for postpartum depressive symptomatology [34], has been validated with standardized psychiatric interviews, and has well-established reliability [35].

\section{Exclusive Breastfeeding}

Exclusive breastfeeding was evaluated by maternal report in the 12 week questionnaire; mothers classified how they were breastfeeding according to the World Health Organization definitions [36].

\section{DATA ANALYSIS}

Data were entered into Access using double data entry with built-in logic and range checks. Descriptive statistics (means, standard deviations, proportions) were used to describe demographic and baseline variables and plans for and actual ISL across time points. Analysis of variance was 
Table 1. Participant Characteristics at Enrollment

\begin{tabular}{|c|c|}
\hline$(n=246)$ & mean \pm SD \\
\hline \multirow[t]{2}{*}{ Gestational age (weeks) at delivery } & $39.3 \pm 1.1$ \\
\hline & $\mathrm{n}(\%)$ \\
\hline \multicolumn{2}{|l|}{ Education completed } \\
\hline University or college & $222(90.2)$ \\
\hline Married/stable Relationship & $239(97.2)$ \\
\hline \multicolumn{2}{|l|}{ Racial background } \\
\hline Black & $17(6.9)$ \\
\hline Multiracial & $12(4.9)$ \\
\hline \multicolumn{2}{|l|}{ Mode of delivery } \\
\hline Spontaneous & $110(44.7)$ \\
\hline Assisted & $36(14.6)$ \\
\hline Caesarean & $100(40.7)$ \\
\hline \multicolumn{2}{|l|}{ EPDS } \\
\hline$>9$ & $58(23.6)$ \\
\hline$>12$ & $13(5.3)$ \\
\hline Partial breastfeeding & $20(8.1)$ \\
\hline Bottle feeding & $2(0.8)$ \\
\hline Unknown & $1(0.4)$ \\
\hline
\end{tabular}

EPDS refers to Edinburgh Postnatal Depression Scale (score range 0-30, score $>12$ indicates at risk for postpartum depression); GSDS refers to General Sleep Disturbance Scale (score range, $0-147$, score $>42$ indicates poor sleeper).

used for continuous variables and chi-square for categorical variables to test for differences between ISL groups on factors that might influence ISL decisions as well as maternal and infant health outcomes.

\section{RESULTS}

In 10 months of recruiting (March 2008 to December 2008), 958 women were approached for consent and 246 agreed to participate and were randomly assigned. Women in the TIPS Trial were overwhelmingly partnered (97\%), had attained post-secondary education $(90 \%)$, and had a mean age of 32 years. The group was racially diverse with $35 \%$ of women identifying themselves as non-Caucasian. Few women had EPDS scores indicating significant depressive symptomatology, most attained GSDS scores $>42$ indicating poor sleep in the last weeks of pregnancy, and the large majority (91\%) planned to exclusively breastfeed their infants. Cesarean section rates were higher in the control group (46\%) than the SI group (35\%); overall rates are higher than the general postpartum population as longer length of stay for women who experienced cesarean delivery allowed for increased opportunity for recruitment. The intervention and control groups were similar on all other baseline and demographic variables and so participant characteristics are presented in aggregate form (Table 1). Outcomes data were available for the large majority of participants $(\mathrm{n}=212$ for 6-week questionnaires and actigraphy; $n=206$ for 12 -week questionnaires, $n=205$ for 12 week actigraphy).

In the immediate postpartum, most women (65\%) planned RS with their infant, while the next most common plan was to use both BS and RS (22\%) (Table 2). The most 
Table 2. Infant Sleep Location Plans in Immediate Postpartum

\begin{tabular}{|l|c|}
\hline \multicolumn{1}{|c|}{$(\mathbf{n = 2 4 6 )}$} & $\mathbf{n}(\mathbf{\%})$ \\
\hline \hline Bed sharing \& room sharing & $53(21.5)$ \\
Bed sharing only & $12(4.9)$ \\
Room sharing only & $161(65.5)$ \\
Neither & $14(5.7)$ \\
Unknown/missing & $6(2.4)$ \\
\hline
\end{tabular}

Table 3. Usual Infant Sleep Location at 6 and 12 Weeks Postpartum

\begin{tabular}{|c|c|c|}
\hline $\mathbf{( n = 2 4 6 )}$ & $\mathbf{6}$ Weeks & $\mathbf{1 2}$ Weeks \\
\hline & $\mathbf{n}(\mathbf{\%})$ & $\mathbf{n}(\mathbf{\%})$ \\
\hline \hline Bed sharing & $41(16.7)$ & $30(12.2)$ \\
Room sharing & $112(45.5)$ & $95(38.6)$ \\
Solitary sleeping & $51(20.7)$ & $70(28.5)$ \\
Unknown/missing & $42(17.1)$ & $51(20.7)$ \\
\hline
\end{tabular}

Table 4. Any Bedsharing at 6 and12 Weeks Postpartum

\begin{tabular}{|c|c|c|}
\hline$(\mathbf{n}=\mathbf{2 4 6})$ & $\mathbf{6}$ weeks & $\mathbf{1 2}$ weeks \\
\hline & $\mathbf{n}(\mathbf{\%})$ & $\mathbf{n}(\mathbf{\%})$ \\
\hline \hline Yes & $125(50.8)$ & $101(41.1)$ \\
No & $86(35.0)$ & $104(42.3)$ \\
Unknown/missing & $35(14.2)$ & $(16.7)$ \\
\hline
\end{tabular}

Table 5. Plans for Infant Sleep Location by Usual Infant Sleep Location at 6 Weeks Postpartum

\begin{tabular}{|l|c|c|c|}
\hline \multicolumn{1}{|c|}{ Infant Sleep Location Plans in Immediate Postpartum } & \multicolumn{2}{c|}{ Usual Infant Sleep Location at 6 Weeks Postpartum } \\
\hline \hline $\begin{array}{l}\text { Bed sharing \& room sharing (n, \%) } \\
(\mathrm{n}=46)\end{array}$ & Bed Sharing & Room Sharing & Solitary Sleeping \\
\hline $\begin{array}{l}\text { Bed sharing only (n, \%) } \\
(\mathrm{n}=7)\end{array}$ & $12(26.1)$ & $3(56.5)$ & $8(17.4)$ \\
\hline $\begin{array}{l}\text { Room sharing only (n, \%) } \\
(\mathrm{n}=134)\end{array}$ & $21(57.1)$ & $81(60.4)$ & $0(0)$ \\
\hline $\begin{array}{l}\text { Neither bed sharing nor room sharing (n, \%) } \\
(\mathrm{n}=13)\end{array}$ & $2(15.4)$ & $0(0)$ & $32(23.9)$ \\
\hline
\end{tabular}

common usual ISL was RS, with $46 \%$ of infants RS at 6 weeks and $39 \%$ at 12 weeks (Table 3). Usually BS was more common than women anticipated in the immediate postpartum; at 6 weeks $17 \%$ of families were BS and $12 \%$ at 12 weeks. BS to any extent was quite common at 6 weeks (51\%) and 12 weeks (41\%) postpartum (Table 4).

Most women were using their planned ISL at 6 weeks postpartum but across all planned ISL categories there were women using different ISL than they expected (Table 5). Although most women remained consistent with usual ISL at 6 and 12 weeks postpartum, there were conversions to a different usual ISL at 12 weeks across all categories of usual ISL at 6 weeks (Table 6). However, it is of note that women whose usual ISL at 6 weeks was RS or SS rarely converted to $\mathrm{BS}$ at 12 weeks.
At 6 and 12 weeks, there were no statistically significant differences between ISL groups in availability of help for infant care at night, or number of other adults in the home (Tables 7 and 8). At both 6 and 12 weeks, there were statistically significant differences in ISL for Caucasian and non-Caucasian mothers, with more BS and little SS in nonCaucasian mothers.

At 6 weeks, usually BS mothers had shorter stretches of sleep than those usually SS (130 mins vs 156 mins; $p=0.03$ ) and more awakenings than those usually RS and SS (11.2 vs 8.9 vs $8.3 ; p=0.001$ ) (Table 9). There were no significant differences between ISL groups on other outcomes at 6 weeks.

At 12 weeks, usually RS mothers had shorter stretches of sleep than those usually SS (164 mins vs 192 mins; $p=0.04$ ) 
Table 6. Usual Infant Sleep Location at 6 Weeks Postpartum by Usual Infant Sleep Location at 12 Weeks Postpartum

\begin{tabular}{|c|c|c|c|}
\hline Usual Infant Sleep Location at 6 Weeks Postpartum & \multicolumn{2}{|c|}{ Usual infant Sleep Location at 12 Weeks Postpartum } \\
\hline \hline $\begin{array}{c}\text { Bed sharing (n, \%) } \\
(\mathrm{n}=32)\end{array}$ & Bed Sharing & $22(68.8)$ & $8(25.0)$ \\
\hline $\begin{array}{c}\text { Room sharing (n, \%) } \\
(\mathrm{n}=102)\end{array}$ & $3(2.9)$ & $77(75.5)$ & $2(6.3)$ \\
\hline $\begin{array}{c}\text { Solitary sleeping(n, \%) } \\
(\mathrm{n}=49)\end{array}$ & $1(2.0)$ & $5(10.2)$ & $43(87.8)$ \\
\hline
\end{tabular}

Table 7. Factors that may Influence Choice of Infant Sleep Location at 6 Weeks Postpartum

\begin{tabular}{|c|c|c|c|c|}
\hline & $\begin{array}{l}\text { Bed Sharing } \\
\qquad(n=41)\end{array}$ & $\begin{array}{c}\text { Room Sharing } \\
(\mathbf{n}=112)\end{array}$ & $\begin{array}{l}\text { Solitary Sleeping } \\
\qquad(\mathrm{n}=51)\end{array}$ & $p$ value \\
\hline & n $(\%)$ & n (\%) & n $(\%)$ & \\
\hline \multicolumn{5}{|l|}{ Racial background } \\
\hline Caucasian & $19(14.1)$ & $70(51.9)$ & $46(34.1)$ & $<0.0001$ \\
\hline Non-caucasian & $21(32.8)$ & $40(62.5)$ & $3(4.7)$ & \\
\hline Hispanic & $1(2.5)$ & $4(3.6)$ & $0(0)$ & \\
\hline Black & $3(7.5)$ & $10(9.1)$ & $0(0)$ & \\
\hline Multiracial & $4(10.0)$ & $4(3.6)$ & $1(2.1)$ & \\
\hline Help with infant care at night & $26(63.4)$ & $65(58.0)$ & $24(47.1)$ & 0.25 \\
\hline Number of other adults in home (mean \pm SD) & $1.38(0.87)$ & $1.24(0.63)$ & $1.07(0.33)$ & 0.076 \\
\hline
\end{tabular}

Table 8. Factors that may Influence Choice of Infant Sleep Location at 12 weeks Postpartum

\begin{tabular}{|c|c|c|c|c|}
\hline & $\begin{array}{l}\text { Bed Sharing } \\
\qquad(n=41)\end{array}$ & $\begin{array}{l}\text { Room Sharing } \\
\quad(n=112)\end{array}$ & $\begin{array}{l}\text { Solitary Sleeping } \\
\qquad(\mathrm{n}=\mathbf{5 1})\end{array}$ & $p$ value \\
\hline & n $(\%)$ & n $(\%)$ & n $(\%)$ & \\
\hline \multicolumn{5}{|l|}{ Racial background } \\
\hline Caucasian & $15(11.3)$ & $58(43.6)$ & $60(45.1)$ & $<0.0001$ \\
\hline Non-caucasian & $14(24.1)$ & $37(63.8)$ & $7(12.1)$ & \\
\hline Hispanic & $0(0)$ & $6(6.3)$ & $0(0)$ & \\
\hline Black & $2(6.9)$ & $8(8.4)$ & $1(1.5)$ & \\
\hline Multiracial & $3(10.3)$ & $5(5.3)$ & $0(0)$ & \\
\hline Help with infant care at night & $13(43.3)$ & $42(45.2)$ & $41(58.6)$ & 0.18 \\
\hline Number of other adults in home (mean \pm SD) & $1.37(0.76)$ & $1.26(0.66)$ & $1.09(0.38)$ & 0.063 \\
\hline
\end{tabular}

(Table 10). There were no significant differences between ISL groups on other outcomes at 12 weeks.

\section{DISCUSSION}

Uptake of the public health recommendation for RS with one's infant is evident in the prevalence $(65.5 \%)$ of newly postpartum women's plans to RS; however, many women also had plans for BS and SS. When usual ISL was examined at 6 and 12 weeks, RS was the most common ISL, with rates declining from 6 to 12 weeks postpartum. BS and SS were not uncommon, with over a quarter of our sample using an
ISL other than RS. Rates of any BS and usual BS in our sample are comparable to those found in other North American studies [5-8, 10]. Although RS remains the most prevalent ISL at 12 weeks postpartum, rates of RS and BS declined from 6 to 12 weeks postpartum, with most families switching to usually having the infant SS. Given that women whose usual ISL at 6 weeks was RS or SS rarely converted to BS at 12 weeks, this may indicate amongst our sample the typically Western value of early acquisition of independent sleep skills, thereby progressing the infant from BS to RS to SS. However, we cannot draw such conclusions from these data without a more detailed investigation of women's 
Table 9. Maternal and Infant Health Outcomes by Infant Sleep Location at 6 Weeks

\begin{tabular}{|c|c|c|c|c|}
\hline & Bed Sharing & Room Sharing & Solitary Sleeping & $p$ value \\
\hline & $\mathrm{n}=\mathbf{4 1}$ & $\mathrm{n}=\mathbf{1 1 2}$ & $\mathrm{n}=\mathbf{5 1}$ & \\
\hline \multicolumn{5}{|l|}{ Maternal sleep } \\
\hline Mean nighttime (9pm-9am) sleep (mins) (SD) & $381(59.9)$ & $390(57.9)$ & $406(49.6)$ & 0.09 \\
\hline Mean longest stretch of nighttime sleep (mins) (SD) & $130(32.6)$ & $150(53.1)$ & $156(35.6)$ & 0.03 \\
\hline Mean number of wakes (SD) & $11.2(3.3)$ & $8.9(3.8)$ & $8.3(3.2)$ & 0.001 \\
\hline Rated own sleep as problem (n, \%) & $15(36.6 \%)$ & $47(42.7 \%)$ & $26(52.0 \%)$ & 0.32 \\
\hline \multicolumn{5}{|l|}{ Infant Sleep } \\
\hline Mean nighttime (9pm-9am) sleep (mins) (SD) & $460(53.8)$ & $461(56.7)$ & $478(51.5)$ & 0.15 \\
\hline Mean longest stretch of nighttime sleep (mins) (SD) & $167(42.9)$ & $175(53.6)$ & $181(55.6)$ & 0.47 \\
\hline Mean number of wakes (SD) & $11(3.2)$ & $11(3.5)$ & $12(3.7)$ & 0.09 \\
\hline Rated infant sleep as problem (n, \%) & $13(31.7 \%)$ & $37(33.3 \%)$ & $11(21.6 \%)$ & 0.31 \\
\hline Mean fatigue, morning, $100 \mathrm{~mm}$ (SD) & $34.0(18.1)$ & $39.5(18.0)$ & $38.9(18.2)$ & 0.25 \\
\hline Mean GSDS Score (SD) & $36.4(15.1)$ & $39.0(16.9)$ & $38.4(17.1)$ & 0.69 \\
\hline Mean EPDS Score (SD) & $4.4(2.8)$ & $5.7(4.1)$ & $5.5(4.2)$ & 0.16 \\
\hline Exclusive breast feeding (n, \%) & $28(68.3 \%)$ & $75(67.6 \%)$ & $39(76.5 \%)$ & 0.50 \\
\hline
\end{tabular}

GSDS refers to General Sleep Disturbance Scale (score range, 0-147, a score $>42$ indicates poor sleeper); EPDS, Edinburgh Postnatal Depression Scale (score range 0-30, score $>12$ indicates at risk for postpartum depression).

Table 10. Maternal and Infant Health Outcomes by Infant Sleep Location at 12 Weeks

\begin{tabular}{|c|c|c|c|c|}
\hline & Bed Sharing & Room Sharing & Solitary Sleeping & $p$ value \\
\hline & $\mathbf{n}=\mathbf{3 0}$ & $\mathrm{n}=\mathbf{9 5}$ & $\mathrm{n}=\mathbf{7 0}$ & \\
\hline \multicolumn{5}{|l|}{ Maternal sleep } \\
\hline Mean nighttime (9pm-9am) sleep (mins) (SD) & $425(62.4)$ & $429(58.1)$ & $445(51.1)$ & 0.14 \\
\hline Mean longest stretch of nighttime sleep (mins) (SD) & $165(85.3)$ & $164(55.4)$ & $192(77.4)$ & 0.04 \\
\hline Rated own sleep as problem (n, \%) & $14(46.7 \%)$ & $35(37.2 \%)$ & $25(35.7 \%)$ & 0.57 \\
\hline \multicolumn{5}{|l|}{ Infant Sleep } \\
\hline Mean nighttime (9pm-9am) sleep (mins) (SD) & $527(58.5)$ & $525(56.2)$ & $530(53.3)$ & 0.82 \\
\hline Mean longest stretch of nighttime sleep (mins) (SD) & $238(71.3)$ & $232(85.0)$ & $242(95.2)$ & 0.75 \\
\hline Mean GSDS Score (SD) & $36.8(17.0)$ & $34.0(18.3)$ & $32.0(15.1)$ & 0.43 \\
\hline Mean EPDS Score (SD) & $5.3(4.4)$ & $4.8(4.0)$ & $4.3(3.5)$ & 0.43 \\
\hline Exclusive breast feeding (n, \%) & $22(75.9 \%)$ & $59(63.4 \%)$ & $49(70.0 \%)$ & 0.40 \\
\hline
\end{tabular}

GSDS refers to General Sleep Disturbance Scale (score range, $0-147$, a score $>42$ indicates poor sleeper); EPDS, Edinburgh Postnatal Depression Scale (score range 0-30, score $>12$ indicates at risk for postpartum depression)

reasons for ISL decisions. Similarly, given that across all planned ISL categories there were women using a different ISL than they expected, further exploration of the events that lead to changes in ISL plans, as well as satisfaction with current ISL, will be important in future research. Parents' experiences with the infant may influence choice of ISL, such that parental responses to infant feeding, crying or sleeping behaviors may shape where the infant sleeps, regardless of prenatal intentions.

Any BS was quite common at both 6 weeks (50.8\%) and 12 weeks (41.1\%); the large number of families who were BS in spite of widely disseminated advice suggest that we 
need to learn much more about parents' decision making process related to ISL. In these future investigations scientific rigor is needed as well as acknowledgment of important cultural influences, personal values and contexts related to these decisions. Parents are faced with a multitude of health information from health care professionals and popular parenting experts that supports, conflicts with, and confuses public health recommendations related to ISL. Further adding complexity to decision-making around ISL is the controversial nature of the evidence around the risks of ISL related to SIDS and accidental infant death. Case-control studies provide evidence regarding practices associated with increased risk of infant death during sleep, but there is much debate over the strength of the evidence and the interaction of risk (e.g. cigarette smoking) and protective factors (e.g. breastfeeding). This debate is often polarized, with the potential risk of a rare, yet tragic event such as SIDS held against the potential benefits of the more common experience of BS and its common companion, breastfeeding.

Few studies have examined women's reasons for choice of ISL. Reasons given for BS include better caregiver and infant sleep, better ability to settle the infant, convenience for infant care and breastfeeding, tradition or parenting philosophy, protection of the child through closer observation of breathing, parent and child emotional needs, and lack of place for the baby to sleep [5, 12, 37]. Reasons for avoiding BS include safety concerns, difficulty in later placing the infant in a crib, and viewing BS as something not traditionally done [5]. Given that we, and others, have found many families are practicing BS with their infants, the concern exists that families are BS, yet are not given information as to how to do so as safely as possible since their choice is counter to recommended practice.

In our sample, ISL location had effects on maternal sleep continuity but was unrelated to other maternal or infant sleep outcomes, maternal fatigue, maternal depressive symptoms or breastfeeding exclusivity. It may be that the differences noted in objective sleep outcomes between ISL were not large enough to result in decrements in subjective evaluations of sleep quality and fatigue or to produce changes in mood. A difference in the mean number of wakes between ISL was only observed at 6 weeks and the differences in longest stretches of sleep were less than 30 minutes; these differences may not be clinically significant. Our findings also suggest that breastfeeding success is unaffected by ISL, as there were no differences between groups in breastfeeding exclusivity. However, it is important to note that our study is the first to examine such relationships, and since ours was a secondary analysis a longitudinal, prospective study should be designed.

Our data have a number of limitations. We did not ask specifically about plans for SS or usual SS and assumed that those who were neither BS nor RS were SS; future studies should include more explicit ISL categories. Our classifications of families as usually BS and RS resulted in a number of cases being set to missing as we could not reliably classify the families as one or the other.

BS was more common and SS less common in our nonCaucasian participants; these findings are in line with those of others $[6,7,9]$. Although our sample was racially diverse, all participants were highly educated and predominantly partnered, limiting our ability to examine effects of SES and social support. Given existing evidence that BS is more common in women without partners and women of low SES [5-8], future research should include such women.

Although we asked women if their infants fell asleep while being fed, rocked or held, the phrasing of the question did not allow us to determine frequency of use of the strategy, thereby missing the opportunity to examine the relationship between usual settling strategies and ISL. BS parents may be more likely to be involved in their child's falling asleep given their close proximity and the child may be less likely to develop "self-soothing" techniques [18, 38, 39]. Given recent evidence that parental involvement in infant sleep initiation may be more important to the development and maintenance of sleep problems than ISL [40], this relationship merits further exploration. Finally, we did not collect outcomes data from the partners of our enrolled women and so examination of the effects of ISL on partners' sleep remains non-existent. Women tend to bear primary responsibility for child care at night [41], which explains our and others' focus on maternal views but leaves little understanding of the partner's sleep experiences or influence on ISL decisions.

Our data suggest there is variation in ISL choice, and ISL changes across the first three months postpartum. Given our findings that ISL has effects on objective maternal sleep outcomes, further prospective, longitudinal research on the effects of ISL on health outcomes is needed to overcome limitations of the secondary analysis approach of our work. Specifically, attention should be given to parents' reasons for ISL choice, a more detailed determination of prevalence of various ISL, demographic predictors of choices, and the relationship to objective and subjective sleep outcomes for the infant, mother, and partner, as well as other health outcomes including breastfeeding, infant crying, satisfaction with partner relationship, and depression across the first postpartum year. Since ISL choice is a controversial one, presenting challenges both to parents and health care practitioners, rigorous work in this area is needed in order to generate high-quality evidence for public health approaches that take into account families' values and beliefs as well as important health effects.

\section{CONFLICT OF INTEREST}

The authors confirm that this article content has no conflicts of interest.

\section{ACKNOWLEDGEMENTS}

Funding for the study was provided by the Canadian Institutes of Health Research (CIHR) (MCT 84658). We are indebted to the families who participated in the study. We are grateful to the staff of the Postpartum Units at Sunnybrook Health Sciences Centre and St. Michael's Hospital for their assistance with the study. Dr. Stremler is recipient of a CIHR New Investigator Award and an Ontario Ministry of Research and Innovation Early Researcher Award.

\section{REFERENCES}

[1] Moon RY. SIDS and other sleep-related infant deaths: expansion of recommendations for a safe infant sleeping environment. Pediatrics 2011; 128(5): 1030-9. 
[2] Recommendations for safe sleeping environments for infants and children. Paediatr Child Health 2004; 9(9): 659-72.

[3] McKenna JJ, McDade T. Why babies should never sleep alone: a review of the co-sleeping controversy in relation to SIDS, bedsharing and breast feeding. Paediatr Respir Rev 2005; 6(2): 134-52.

[4] Thoman EB. Co-sleeping, an ancient practice: issues of the past and present, and possibilities for the future. Sleep Med Rev 2006; 10(6): 407-17.

[5] Hauck FR, Signore C, Fein SB, Raju TN. Infant sleeping arrangements and practices during the first year of life. Pediatrics 2008; 122 Suppl 2: S113-20.

[6] Lahr MB, Rosenberg KD, Lapidus JA. Maternal-infant bedsharing: risk factors for bedsharing in a population-based survey of new mothers and implications for SIDS risk reduction. Matern Child Health J 2007 ; 11(3): 277-86.

[7] Willinger M, Ko CW, Hoffman HJ, Kessler RC, Corwin MJ. Trends in infant bed sharing in the United States, 1993-2000: the National Infant Sleep Position study. Arch Pediatr Adolesc Med 2003; 157(1): 43-9.

[8] McCoy RC, Hunt CE, Lesko SM, et al. Frequency of bed sharing and its relationship to breastfeeding. J Dev Behav Pediatr. 2004; 25(3): 141-9.

[9] Fu LY, Colson ER, Corwin MJ, Moon RY. Infant sleep location: associated maternal and infant characteristics with sudden infant death syndrome prevention recommendations. J Pediatr 2008; 153(4): 503-8.

[10] Ateah CA, Hamelin KJ. Maternal bedsharing practices, experiences, and awareness of risks. J Obstet Gynecol Neonatal Nurs. 2008; 37(3): 274-81.

[11] Blair PS, Ball HL. The prevalence and characteristics associated with parent-infant bed-sharing in England. Arch Dis Child 2004; 89(12): 1106-10.

[12] Ball H. Reasons to bed-share: why parents sleep with their infants. J Reprod Infant Psychol 2002; 20(4): 207-21.

[13] Gay CL, Ward TM, Towns SL, Lee KA. Parent-newborn cosleeping in the San Francisco Bay area. Sleep 2004; 27(Abstract supplement): A411.

[14] Gay CL, Lee KA, Lee SY. Sleep patterns and fatigue in new mothers and fathers. Biol Res Nurs 2004; 5(4): 311-8.

[15] Elek SM, Hudson DB, Fleck MO. Expectant parents' experience with fatigue and sleep during pregnancy. Birth 1997; 24(1): 49-54.

[16] Dinges DF, Pack F, Williams K, et al. Cumulative sleepiness, mood disturbance, and psychomotor vigilance performance decrements during a week of sleep restricted to 4-5 hours per night. Sleep. 1997; 20(4): 267-77.

[17] Stepanski EJ. The effect of sleep fragmentation on daytime function. Sleep $2002 ; 25(3): 268-76$.

[18] Touchette E, Petit D, Paquet J, et al. Factors associated with fragmented sleep at night across early childhood. Arch Pediatr Adolesc Med 2005; 159(3): 242-9.

[19] Mosko S, Richard C, McKenna J. Maternal sleep and arousals during bedsharing with infants. Sleep 1997; 20(2): 142-50.

[20] Mosko S, Richard C, McKenna J, Drummond S. Infant sleep architecture during bedsharing and possible implications for SIDS. Sleep 1996; 19(9): 677-84.

[21] Mao A, Burnham MM, Goodlin-Jones BL, Gaylor EE, Anders TF. A comparison of the sleep-wake patterns of cosleeping and solitary-sleeping infants. Child Psychiatry Hum Dev 2004; 35(2): 95-105.

[22] DeLeon CW, Karraker KH. Intrinsic and extrinsic factors associated with night waking in 9-month-old infants. Infant Behav Dev 2007; 30(4): 596-605.

[23] Dennis CL, Ross L. Relationships among infant sleep patterns, maternal fatigue, and development of depressive symptomatology. Birth 2005; 32(3): 187-93.

[24] Dorheim SK, Bondevik GT, Eberhard-Gran M, Bjorvatn B. Sleep and depression in postpartum women: a population-based study. Sleep 2009; 32(7): 847-55.

[25] Skouteris H, Germano C, Wertheim EH, Paxton SJ, Milgrom J. Sleep quality and depression during pregnancy: a prospective study. J Sleep Res 2008; 17(2): 217-20.

[26] Ford DE, Kamerow DB. Epidemiologic study of sleep disturbances and psychiatric disorders. An opportunity for prevention? JAMA 1989; 262(11): 1479-84.

[27] Krystal AD. Depression and insomnia in women. Clin Cornerstone. 2004; 6 Suppl 1B: S19-28.

[28] Stremler R, Hodnett E, Kenton L, et al. Effect of behaviouraleducational intervention on sleep for primiparous women and their infants in early postpartum: multisite randomised controlled trial. BMJ 2013; 346f1164. doi: http://dx.doi.org/10.1136/bmj.f1164.

[29] Littner M, Kushida CA, Anderson WM, et al. Practice parameters for the role of actigraphy in the study of sleep and circadian rhythms: an update for 2002. Sleep 2003; 26(3): 337-41.

[30] Lee KA, Hicks G, Nino-Murcia G. Validity and reliability of a scale to assess fatigue. Psychiatry Res 1991; 36(3): 291-8.

[31] Waters MA, Lee KA. Differences between primigravidae and multigravidae mothers in sleep disturbances, fatigue, and functional status. J Nurse Midwifery 1996; 41(5): 364-7.

[32] Lee KA. Self-reported sleep disturbances in employed women. Sleep 1992; 15(6): 493-8.

[33] Cox JL, Holden JM, Sagovsky R. Detection of postnatal depression. Development of the 10-item Edinburgh Postnatal Depression Scale. Br J Psychiatry 1987; 150: 782-6.

[34] Beck CT. Predictors of postpartum depression: an update. Nurs Res 2001; 50(5): 275-85.

[35] Affonso DD, De AK, Horowitz JA, Mayberry LJ. An international study exploring levels of postpartum depressive symptomatology. J Psychosom Res 2000; 49(3): 207-16.

[36] Labbok M, Krasovec K. Toward consistency in breastfeeding definitions. Stud Fam Plann. 1990; 21(4): 226-30.

[37] Chianese J, Ploof D, Trovato C, Chang JC. Inner-city caregivers' perspectives on bed sharing with their infants. Acad Pediatr 2009; 9(1): 26-32.

[38] Anders TF. Night-waking in infants during the first year of life. Pediatrics 1979; 63(6): 860-4.

[39] Goodlin-Jones BL, Burnham MM, Gaylor EE, Anders TF. Night waking, sleep-wake organization, and self-soothing in the first year of life. J Dev Behav Pediatr 2001; 22(4): 226-33.

[40] Mindell JA, Sadeh A, Kohyama J, How TH. Parental behaviors and sleep outcomes in infants and toddlers: a cross-cultural comparison. Sleep Med 2010; 11(4): 393-9.

[41] Venn S, Arber S, Meadows R, Hislop J. The fourth shift: exploring the gendered nature of sleep disruption among couples with children. Br J Soc 2008; 59(1): 79-97. 